\title{
O espectro clínico e radiológico da pneumonia em organização: análise retrospectiva de 38 casos* $^{*}$
}

\author{
Fabrício Piccoli Fortuna ${ }^{1}$, CRistiano Perin ${ }^{2}$, Juliano De Bortoli², Geraldo Resin GeyeR ${ }^{3}$, \\ NELSON DA SILVA PORTO ${ }^{4}$, AdALBERTO SPERB RUBIN ${ }^{5}$
}

A pneumonia em organização, acompanhada ou não de bronquiolite obliterante, é uma condição anatomopatológica pulmonar específica com uma grande variedade de apresentações clínicas e radiológicas. A pneumonia em organização pode ser idiopática ou secundária a várias doenças, incluindo infecções e drogas. Objetivos: Descrever as manifestações clínicas, dados radiológicos e espirométricos de pacientes com pneumonia em organização. Métodos: Análise retrospectiva de pacientes com o diagnóstico de pneumonia em organização. Resultados: 38 pacientes foram incluídos na análise. Quatorze apresentavam também condições clínicas relacionadas à pneumonia em organização, e estes mais freqüentemente apresentaram infiltração pulmonar difusa (três de quatro

casos) e bronquiolite obliterante associada ( $57 \%$ vs. $20 \%, \mathrm{p}=0,05)$. Dos 13 pacientes com bronquiolite obliterante associada apenas um era assintomático e dois apresentavam lesões localizadas

(15\%). De oito pacientes assintomáticos com lesões localizadas, nenhum possuía bronquiolite obliterante associada, e a apresentação radiológica freqüentemente mimetizou carcinoma brônquico. A espirometria de modo geral não contribuiu sobremaneira para o diagnóstico, provavelmente devido

à alta prevalência de tabagismo na amostra. Conclusões: A pneumonia em organização possui apresentação clínica e radiológica variada. Fatores que sugerem a ausência de bronquiolite obliterante são a ausência de sintomas e lesões radiológicas localizadas, e esta forma da doença mais freqüentemente deve ser diferenciada de carcinoma brônquico na prática clínica.

(J Pneumol 2002;28(6):317-23)

\section{Clinical and radiographic spectrum of organizing pneumonia: retrospective analysis of 38 cases}

Organizing pneumonia, whether or not accompanied by bronchiolitis obliterans, is a specific anatomicopathological condition of the lungs that can present in a variety of clinical and radiographic ways. It can be either idiopathic or secondary to a number of diseases, including infection and drugs. Objectives: To describe the clinical manifestations, radiographic and spirometric data seen in patients with organizing pneumonia. Methods: Retrospective analysis of patients with a diagnosis of organizing pneumonia. Results: 38 patients were included in the analysis. Fourteen also had clinical conditions related to organizing pneumonia, and they presented more frequently with diffuse pulmonary infiltrates (three of four cases) and associated bronchiolitis obliterans (57\% vs. $20 \%, p=0,05)$. Of the 13 patients with bronchiolitis obliterans, only one was asymptomatic and two had localized lesions (15\%). Of the eight asymptomatic patients with localized lesions, no one had associated bronchiolitis obliterans, and the radiographic appearance often resembled bronchial carcinoma. Spirometry was generally of little

* Trabalho realizado no Pavilhão Pereira Filho - ISCMPA. Fundação Faculdade Federal de Ciências Médicas de Porto Alegre (FFFCMPA).

1. Médico Residente em Pneumologia da FFFCMPA - Pavilhão Pereira Filho.

2. Acadêmico de Medicina (FFFCMPA).

3. Patologista do Pavilhão Pereira Filho (ISCMPA). Responsável pelo Serviço de Anatomia Patológica do Hospital Ernesto Dornelles.
4. Radiologista e Diretor Médico do Pavilhão Pereira Filho (ISCMPA). Professor do Programa de Pós-Graduação em Pneumologia pela Universidade do Rio Grande do Sul.

5. Doutor em Pneumologia pela UFRGS. Médico Pneumologista do Pavilhão Pereira Filho (ISCMPA). Professor da FEEVALE (RS).

Endereço para correspondência - Adalberto Sperb Rubin, Rua Almirante Abreu, 246/402 - 90420-010 - Porto Alegre, RS. E-mail: arubin@terra.com.br

Recebido para publicação em 4/3/02. Aprovado, após revisão, em 18/8/02. 
value to diagnosis, probably because of the high prevalence of smoking in the sample.

Conclusion: The clinical and radiographic presentation of organizing pneumonia is variable.

Factors that suggest the absence of coexisting bronchiolitis obliterans are the absence of symptoms and localized radiographic lesions, and this form of the disease has to be more often differentiated from bronchial carcinoma in clinical practice.

Descritores - Bronquiolite obliterante. Pneumonia por bronquiolite obliterante em organização. Doenças pulmonares intersticiais.

Key words - Bronchiolitis obliterans. Interstitial lung diseases. Bronchiolitis obliterans in organizing pneumonia.

\section{INTRODUÇÃO}

A pneumonia em organização (PO) é uma entidade anatomopatológica caracterizada pelo acúmulo de tecido de granulação na luz das vias aéreas terminais, que progride de um exsudato fibrinoso para uma matriz colagênica frouxa contendo fibroblastos. Esse acúmulo acomete principalmente os alvéolos, mas pode associar-se a comprometimento concomitante do bronquíolo respiratório, configurando uma bronquiolite obliterante. A pneumonia em organização, com ou sem bronquiolite obliterante (BO), não é um achado específico, mas reflete um padrão de injúria pulmonar que pode ser secundário a diversas causas, como infecções, colagenoses, radioterapia e uso de fármacos. A BO pode também ser uma entidade clínica específica, de etiologia indeterminada, quando então é chamada de idiopática ou criptogênica ${ }^{(1-3)}$. O outro termo utilizado é o de bronquiolite obliterante com pneumonia em organização (BOOP, do inglês bronchiolitis obliterans organising pneumonia), proposto por Epler et al. na série de casos que descreve a forma idiopática ${ }^{(4)}$. Esse termo, no entanto, tem sido cada vez menos empregado por enfatizar demasiadamente o componente bronquiolar, que pode ser encontrado em uma variedade de afecções não necessariamente associadas à pneumonia em organização(5).

A distinção entre a forma criptogênica e a secundária de pneumonia em organização é difícil de ser estabelecida com segurança através de dados clínicos ou radiológicos, sendo a presença de doenças e condições associadas o dado que sugere a possibilidade de causalidade. Entretanto, tais condições coexistem freqüentemente no mesmo paciente, dificultando o diagnóstico etiológico. A maioria dos autores inclui apenas casos idiopáticos, simplificando o real espectro da pneumonia em organização. Este trabalho sumariza as características clínicas $e$ radiológicas de 38 casos de pneumonia em organização, comparando-as entre os pacientes agrupados de acordo com os sintomas observados e a presença de doenças associadas.
Siglas e abreviaturas utilizadas neste trabalho

PO - Pneumonia em organização

$\mathrm{BO}$ - Bronquiolite obliterante

BOOP - Bronquiolite obliterante com pneumonia em organização

$\mathrm{VEF}_{1}$ - Volume expiratório forçado no primeiro segundo

CVF - Capacidade vital forçada

CPT - Capacidade pulmonar total

VR - Volume residual

DCO - Difusão para o monóxido de carbono

SDRA - Síndrome do desconforto respiratório do adulto

SIDA - Síndrome da imunodeficiência humana adquirida

HIV -

\section{MÉTOdOS}

Após uma busca pelo termo pneumonia em organização em um banco de dados de descrições anatomopatológicas de biópsias pulmonares no período de 1993 a 2001, foram identificados 42 pacientes nos quais o achado de pneumonia em organização foi considerado o diagnóstico primário. Os prontuários médicos desses pacientes foram, então, retrospectivamente revisados e os seguintes dados sistematicamente registrados: idade, sexo, tabagismo, sintomas, apresentação radiológica, resultados de provas de função pulmonar, método de obtenção do espécime pulmonar e presença de bronquiolite obliterante na histologia. Quatro pacientes foram excluídos da análise por falta de informações adequadas sobre a doença. Todos os pacientes realizaram radiografias convencionais de tórax e 25 (65\%) também foram submetidos a avaliação tomográfica computadorizada de tórax. As biópsias pulmonares foram obtidas por toracotomia em 22 casos; transtorácica com agulha lancetante em 11 casos; fibrobroncoscopia com biópsia transbrônquica em quatro casos; e necropsia em um caso.

Dados espirométricos estavam disponíveis para análise em 22 pacientes. Volume expiratório forçado no primeiro segundo $\left(\mathrm{VEF}_{1}\right)$, capacidade vital forçada (CVF) e demais fluxos expiratórios foram obtidos em todos os pacientes. Medidas da capacidade pulmonar total (CPT) e volume residual (VR) utilizando o método da diluição do hélio foram obtidos em 11 pacientes e em 10 foi mensurado o coeficiente de difusão para o monóxido de carbono (DCO) utilizando o método de respiração única. Todos os dados foram obtidos através do espirômetro Collins GS plus do Laboratório de Função Pulmonar do Pavilhão Pereira Filho. 
TABELA 1

Características clínicas dos pacientes com pneumonia em organização, estratificados por etiologia e presença de bronquiolite obliterante

\begin{tabular}{|c|c|c|c|c|c|}
\hline & $\begin{array}{c}\text { Total } \\
(\mathbf{N}=38)\end{array}$ & $\begin{array}{c}\text { PO } \\
\text { criptogênica } \\
(\mathrm{N}=\mathbf{2 4 )}\end{array}$ & $\begin{array}{c}\text { PO } \\
\text { secundários } \\
(\mathrm{N}=14)\end{array}$ & $\begin{array}{c}\text { Casos } \\
\text { sem BO } \\
(N=25)\end{array}$ & $\begin{array}{c}\text { Casos } \\
\text { com BO } \\
(N=13)\end{array}$ \\
\hline Idade (média) & 57 & 59 & 53 & 58 & 54 \\
\hline Sexo masculino (\%) & $22(57,9 \%)$ & $13(54,2 \%)$ & $9(64,3 \%)$ & $17(68 \%)$ & $5(38 \%)$ \\
\hline D ispnéia (\%) & $19(50 \%)$ & $7(29,1 \%)$ & $8(57,1 \%)$ & $11(44 \%)$ & $8(61,5 \%)$ \\
\hline Astenia (\%) & $9(23,7 \%)$ & $3(12,5 \%)$ & $3(21,4 \%)$ & $6(24 \%)$ & $3(23,1 \%)$ \\
\hline Febre $(\%)$ & $13(34,2 \%)$ & $7(29,1 \%)$ & $6(42,8 \%)$ & $8(32 \%)$ & $5(38,5 \%)$ \\
\hline Tosse (\%) & $24(63,2 \%)$ & $18(75 \%)^{*}$ & $6(42,8 \%)$ & $15(60 \%)$ & $9(69,2 \%)$ \\
\hline Expectoração (\%) & $10(26,3 \%)$ & $3(12,5 \%)$ & $3(21,4 \%)$ & $5(20 \%)$ & $5(38,5 \%)$ \\
\hline Emagrecimento (\%) & $11(28,9 \%)$ & $4(16,6 \%)$ & $4(28,5 \%)$ & $6(24 \%)$ & $5(38,5 \%)$ \\
\hline Hemoptise (\%) & $3(7,9 \%)$ & $2(8,3 \%)$ & $1(7,1 \%)$ & $2(8 \%)$ & $1(7,6 \%)$ \\
\hline Dor torácica (\%) & $13(34,2 \%)$ & $5(20,8 \%)$ & $2(14,2 \%)$ & $8(32 \%)$ & $5(38,5 \%)$ \\
\hline
\end{tabular}

* $p=0,047$ em comparação com o grupo de casos secundários.

Os 38 pacientes foram comparados de acordo com a presença ou ausência de doença predisponente e presença ou ausência de bronquiolite obliterante associada. As comparações entre os grupos foram realizadas através do teste do qui-quadrado com correção de Yates para as variáveis categóricas e teste $t$ de Student para as variáveis contínuas.

\section{RESULTADOS}

\section{Características gerais}

Foram estudados 38 pacientes que preencheram os critérios de inclusão no estudo. A idade variou de 27 a 76 anos, sendo a média de 57 anos. Vinte e dois pacientes eram do sexo masculino (58\%). Todos os pacientes eram da cor branca. Cinqüenta e cinco por cento dos pacientes eram fumantes atuais ou prévios, com média de consumo de 32 anos/maço (7-90). Sintomas estavam presentes em 26 pacientes (68\%), sendo tosse o sintoma mais comum (63\% dos casos). A tosse era produtiva em $41 \%$ dos casos. Dispnéia, aos esforços e geralmente de caráter progressivo, foi referida por $50 \%$ dos pacientes. Febre e dor torácica estavam presentes em $34 \%$, enquanto astenia e emagrecimento em $23 \%$ e $29 \%$ dos casos, respectivamente. Hemoptise, já descrita inclusive como sintoma principal(6), foi referida por três pacientes $(8 \%)$. A Tabela 1 sumariza os achados clínicos.

A alteração mais comum ao exame físico foi a redução do murmúrio vesicular na ausculta pulmonar, notada em 16 pacientes ( $42 \%$ ), sendo esta difusa em $56 \%$ dos casos e localizada em $44 \%$. Estertores crepitantes foram auscultados em 10 pacientes (26\%), sendo bilaterais em cinco casos. Nenhum dos achados na ausculta pulmonar foi específico para tipo ou localização das anormalidades ra- diológicas. Taquipnéia estava presente ao repouso em apenas quatro pacientes. Um paciente apresentava hipocratismo digital, sem qualquer doença associada potencialmente determinante desta manifestação.

A Tabela 2 e a Figura 1 mostram a freqüencia das diferentes apresentações radiológicas. Consolidações, únicas ou múltiplas (Figuras 2 e 3), foram as anormalidades mais freqüentes, seguidas de infiltração difusa, geralmente heterogênea e de padrão granular ou em "vidro-fosco", as-

TABELA 2

Freqüência das anormalidades radiológicas

\begin{tabular}{lc}
\hline \multicolumn{1}{c}{ Tipo de apresentação radiológica } & Freqüência \\
\hline Consolidação isolada & 13 \\
Consolidações múltiplas & 9 \\
Infiltrado bilateral & 4 \\
Consolidações múltiplas associadas a infiltrado difuso & 5 \\
N ódulo único & 3 \\
Consolidações associadas a nódulos & 2 \\
N ódulos múltiplos & 1 \\
Infiltrado associado a consolidação isolada & 1 \\
\hline
\end{tabular}

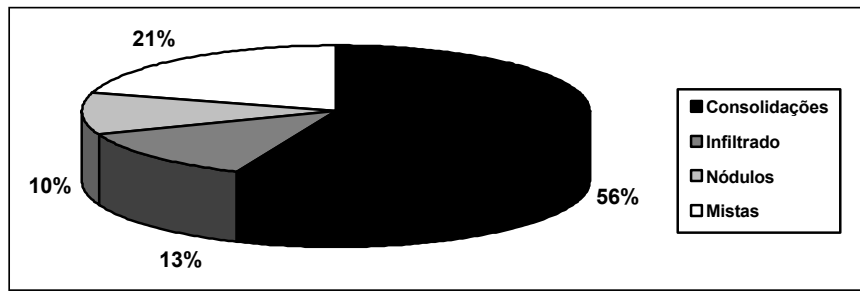

Figura 1 - Freqüência percentual das anormalidades radiológicas agrupadas 


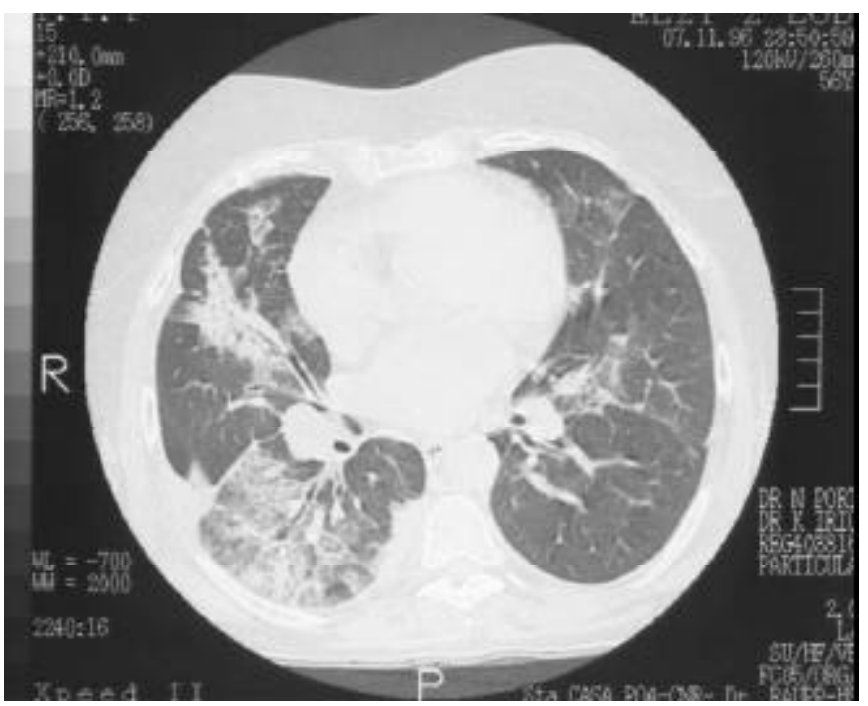

Figura 2 - Tomografia computadorizada de tórax de uma paciente com pneumonia em organização manifestando-se como múltiplas consolidações. Esta paciente possuía BO associada na histopatologia.

sociada ou não a consolidações (Figura 4). Em três casos, o achado radiológico foi um nódulo pulmonar solitário. Seis pacientes apresentavam consolidação com broncograma aéreo e cinco, atelectasia. Derrame pleural foi encontrado em três casos. A Tabela 3 descreve a localização da anormalidades radiológicas.

Dos 22 pacientes submetidos a avaliação espirométrica, em 10 (45\%) os valores encontrados estiveram acima do limite inferior da normalidade. $\mathrm{O} \mathrm{VEF}_{1}$ médio da amostra foi de $76 \%$ do previsto e a CVF média, de $84 \%$ do previsto (Tabela 4). Três pacientes eram portadores de distúrbio restritivo, de acordo com os critérios da Sociedade Brasileira de Pneumologia e Tisiologia( ${ }^{(7)}$, com CVF média de $56 \%$ do previsto. Destes, dois realizaram medida da CPT ( $46 \%$ e $51 \%$ do previsto). Sete pacientes eram portadores de distúrbio obstrutivo e dois de distúrbio misto. Oito dos 10 pacientes que realizaram a medida da DCO tiveram resultados anormais (inferiores a $70 \%$ do previsto), sendo a DCO média destes correspondente a $52 \%$ do previsto (41-65).

Quando analisados os resultados da função pulmonar em não-fumantes (sete casos), apenas um apresentava distúrbio obstrutivo, com redução da CVF. Três pacientes ti-

TABELA 3*

\section{Resultados da avaliação funcional pulmonar}

\begin{tabular}{lccc}
\hline & Consolidações & Nódulos & Total (\%) \\
\hline Lobo superiores e médio & 22 & 6 & $28(62 \%)$ \\
Lobos inferiores & 16 & 1 & $17(38 \%)$ \\
\hline
\end{tabular}

* Refere-se às lesões localizadas, quando havia dados disponíveis.

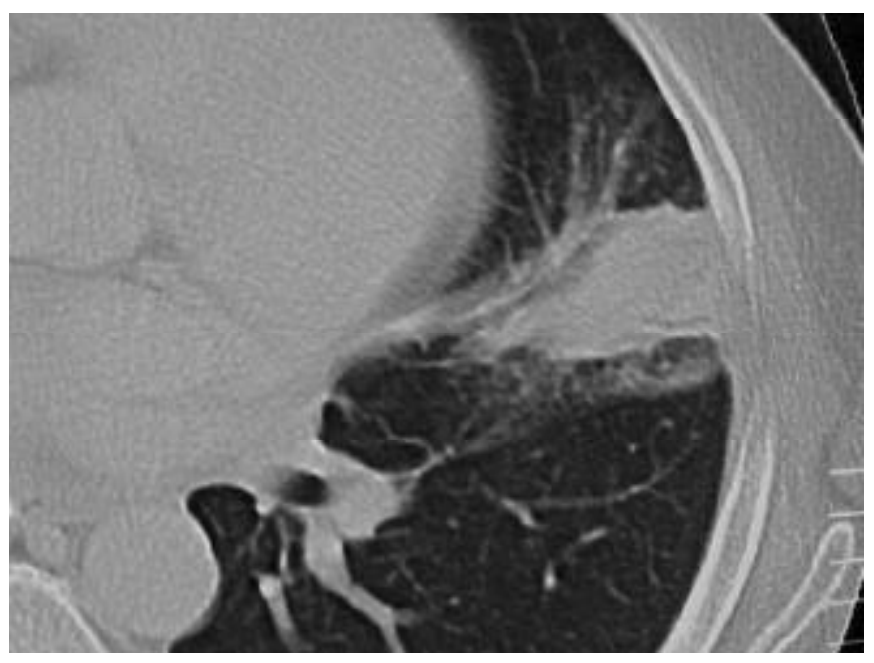

Figura 3 - Tomografia computadorizada de tórax de uma paciente com pneumonia em organização manifestando-se como consolidação única localizada

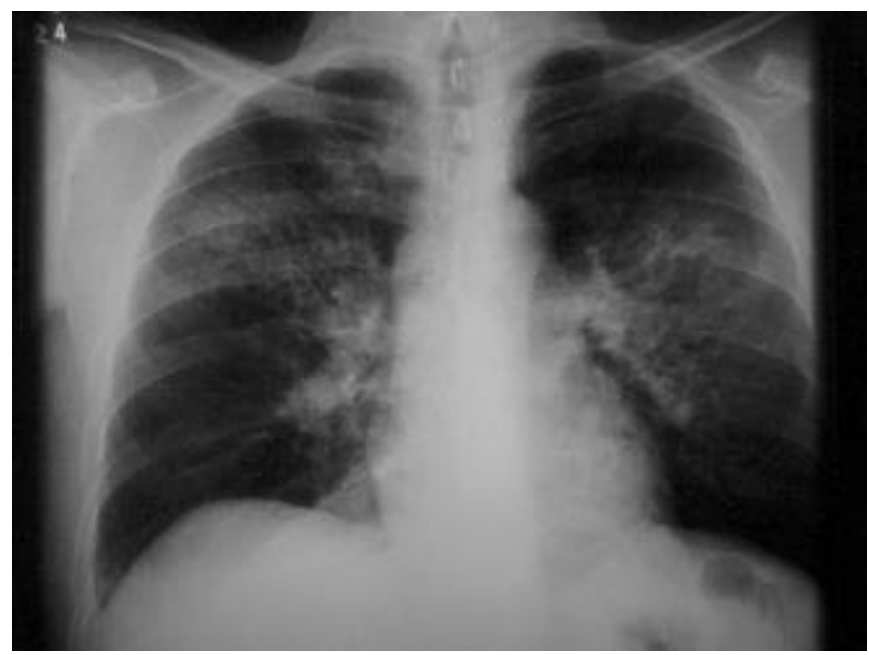

Figura 4 - Radiografia frontal de tórax evidenciando infiltrado bilateral em "vidro-fosco", que em certas áreas assume caráter consolidativo. Este paciente recebeu o diagnóstico de $P O$ criptogênica, não associada a $B O$ na histopatologia.

TABELA $4^{*}$

Localização das anormalidades radiológicas

\begin{tabular}{lrr}
\hline \multicolumn{1}{c}{ Variável } & $\begin{array}{r}\text { Total amostra } \\
\text { Média } \pm \text { DP }\end{array}$ & $\begin{array}{r}\text { Não-fumantes } \\
\text { Média } \pm \text { DP }\end{array}$ \\
\hline CVF (\% previsto) & $84 \% \pm 26 \%$ & $65 \% \pm 20 \%$ \\
VEF $_{1}$ (\% previsto) & $76 \% \pm 29 \%$ & $62 \% \pm 20 \%$ \\
VEF $_{1} /$ CVF (\%) & $70 \% \pm 16 \%$ & $74 \% \pm 14 \%$ \\
CPT $\left(\%\right.$ previsto) $_{\text {VR }} \%$ previsto) & $90 \% \pm 27 \%$ & $59 \% \pm 14 \%$ \\
DCO & $137 \% \pm 61 \%$ & $75 \% \pm 19 \%$ \\
\hline
\end{tabular}

* N enhuma das variáveis atingiu significância estatística na comparação entre os grupos. 
nham distúrbio restritivo; dois, distúrbio misto e um, valores considerados normais. Todos os quatro dentre estes que mensuraram a DCO obtiveram resultados inferiores a $70 \%$ do previsto.

\section{Casos de pneumonia em organização secundária}

Do grupo de 14 pacientes com pneumonia em organização presumivelmente secundária, os sintomas duraram menos do que 30 dias na subtotalidade dos casos. A Tabela 1 resume o quadro clínico desse grupo. As condições associadas foram: dois casos após quimioterapia; dois casos pós-transplante pulmonar; um caso de uso de amiodarona; um caso após pneumonia por Legionella pneumophila, confirmada por anticorpos séricos três meses antes do início dos sintomas atuais, mas também portador de retocolite ulcerativa e usuário de sulfassalazina havia seis anos; um caso de consolidação associada a empiema por bacilos gramnegativos; um caso após radioterapia adjuvante para neoplasia de mama; um caso com história de radioterapia prévia para neoplasia de mama com evolução rápida para síndrome do desconforto respiratório do adulto (SDRA), cuja biópsia mostrava sinais de dano alveolar difuso concomitante; um caso secundário a pneumonia causada por Streptococcus pneumoniae; um caso após transplante renal (27 dias); um caso após pneumonia por Pseudomonas aeruginosa; um caso portador de síndrome da imunodeficiência humana adquirida (SIDA) e um caso após sepse por Staphylococcus aureus resistente à meticilina. Do total de quatro casos em que a lesão radiológica era infiltração pulmonar difusa, três encontravam-se neste subgrupo.

\section{Casos de pneumonia em organização criptogê- nica}

O maior número de pacientes analisados não possuía condições associadas (24 casos). Em $61 \%$ dos pacientes sintomáticos, os sintomas tiveram duração inferior a quatro semanas, com duração média de 10 semanas. Tosse foi um sintoma mais comum nos casos sem etiologia do que nos casos secundários (Tabela 1).
Houve um subgrupo de oito pacientes nos quais o achado radiológico foi incidental. Nódulos ou consolidações localizados, na sua maioria nos lobos superiores (Tabela 3 ), foram os achados radiológicos mais freqüentes. Nenhum deles esteve associado a infiltrações no exame radiológico. Nenhum desses pacientes apresentou estertores crepitantes na ausculta pulmonar ou bronquiolite obliterante no espécime analisado.

Quando comparados os pacientes com pneumonia em organização associada a outras doenças com aqueles sem etiologia definida, os primeiros apresentaram menor freqüência de tosse e maior número de biópsias demonstrando bronquiolite obliterante associada (Tabela 5).

TABELA 5

Comparação entre pacientes com pneumonia em organização secundária e pacientes com pneumonia em organização criptogênica

\begin{tabular}{lccccc} 
Sintomáticos & $11 / 14(77 \%)$ & $15 / 24(62 \%)$ & - & 0,505 & - \\
\hline $\begin{array}{l}\text { Presença de } \\
\text { estertores } \\
\text { crepitantes }\end{array}$ & $3 / 14(21 \%)$ & $7 / 24(29 \%)$ & - & 0,88 & - \\
\hline $\begin{array}{l}\text { Apresentação } \\
\text { radiológica }\end{array}$ & $6 / 14(42 \%)$ & $11 / 24(45 \%)$ & $5 / 8(62 \%)$ & 0,6 & - \\
$\begin{array}{l}\text { localizada } \\
\begin{array}{l}\text { Presença de } \\
\text { bronquiolite } \\
\text { obliterante }\end{array}\end{array}$ & $8 / 14(57 \%)$ & $5 / 24(20 \%)$ & - & 0,055 & 5,06 \\
\hline
\end{tabular}

* Todos incluídos na análise dos casos sem etiologia.
Casos de pneumonia em organização e bronquiolite obliterante

Do total de 38 pacientes, 13 apresentavam bronquiolite obliterante associada à pneumonia em organização. Destes, apenas dois apresentavam alterações radiológicas localizadas. Nove pacientes (69\%) apresentavam consolidações múltiplas, associadas ou não a infiltrado, e dois, infiltrado difuso. Apenas um desses pacientes era assintomático. Oito desses pacientes (quatro eram fumantes) realizaram espirometria e os resultados foram inespecíficos: três foram consideradas normais, três apresentavam distúrbio obstrutivo, um distúrbio restritivo e um distúrbio misto. A maioria (8/13) desses pacientes apresentava condições associadas (Tabela 6).

\section{DisCUSSÃO}

Foram Epler et al. ${ }^{(4)}$ que pela primeira vez distinguiram casos de pneumonia em organização de casos de fibrose 
pulmonar idiopática. $\mathrm{O}$ termo por eles empregado, entretanto, foi bronquiolite obliterante, e só após a organização intraluminal característica tornou-se óbvia. Isso provavelmente reduziu o espectro de pacientes com o diagnóstico; as características clínicas, radiológicas e funcionais da sua amostra de 57 pacientes eram homogêneas, apesar da inclusão de sete casos provavelmente secundários. As características clínicas da presente série são semelhantes, mas os pacientes da série de Epler et al. tinham incidência muito menor de anormalidades radiológicas localizadas (7\% contra $44 \%$ ) e distúrbios ventilatórios obstrutivos na avaliação funcional pulmonar. Como em nossos pacientes, DCO reduzida foi o achado anormal mais freqüente. Outras séries com critérios de inclusão similares relataram achados clínicos e radiológicos semelhantes ${ }^{(8,9)}$.

Cordier et al. ${ }^{(10)}$, em sua série de 16 pacientes sem etiologia definida, encontraram características mais diversificadas. Eles agruparam os pacientes em três grupos de acordo com a apresentação radiológica: quatro apresentaram consolidações múltiplas, cinco, consolidação localizada e sete, infiltrado difuso bilateral, que foi a forma menos comum nos 24 pacientes da série sem etiologia definida. Nessa série, os pacientes com consolidação única apresentaram hemoptise mais freqüentemente, associação não encontrada em nossos casos, mas as lesões localizadas também predominaram nos lobos superiores. Em série de seis casos descritos como idiopáticos, Bellomo et al. ${ }^{(11)}$ igualmente encontraram apresentações clínicas e radiológicas diversas, com apresentações radiológicas localizadas. Em um caso, entretanto, o paciente estava usando amiodarona, uma possível associação.

Houve um grande número de pacientes com lesões localizadas nessa série (19/38). Acreditamos que muitos casos de pneumonia em organização secundária a infecção tenham sido incluídos nessa descrição, principalmente entre os casos assintomáticos, mas provavelmente também entre os sem etiologia definida. Müller et al. ${ }^{(12)}$ encontraram consolidações localizadas, associadas ou não a nódulos, em cinco de um total de 14 pacientes. Outro estudo analisou 18 pacientes com anormalidades localizadas, a maior parte, nodulares. Quinze pacientes eram assintomáticos e os outros três apresentavam tosse produtiva. Não houve descrição da localização das lesões e todos os pacientes submeteram-se a ressecção cirúrgica. Broncograma aéreo e margem irregular foram achados freqüentes, dificultando o diagnóstico diferencial com car-
TABELA 6

nia em organização com e sem bronquiolite obliterante

$\begin{array}{cccc}\text { PO com } & \text { PO sem } & \text { Valor } & \text { RR } \\ \text { BO } & \text { BO } & \text { de } p & \text { (IC 95\%) } \\ (n=13) & (n=25) & & \end{array}$

\begin{tabular}{rrrc}
$12 / 13(92 \%)$ & $13 / 25(52 \%)$ & 0,034 & $11,07(1,24-98,55)$ \\
$6 / 13(46 \%)$ & $7 / 25(28 \%)$ & 0,448 & - \\
$2 / 13(15 \%)$ & $15 / 25(60 \%)$ & 0,023 & $0,12(0,02-0,66)$ \\
\hline
\end{tabular}

cinoma brônquico. A maioria das lesões localizava-se na porção cortical do parênquima pulmonar, na região subpleural, ou ao longo de feixes broncovasculares, e o formato oval ou trapezóide com lesões satélites foi comum. Nos oito casos em que se repetiu a tomografia de tórax, alteração nos achados ocorreu em seis ${ }^{(13)}$. Casos com quadro clínico característico da forma idiopática também podem apresentar-se como nódulos isolados ${ }^{(14)}$, mas o seguimento com exames radiológicos mostra que alguns desses pacientes desenvolvem lesões múltiplas posteriormente. Como pode ser observado, o diagnóstico diferencial entre pneumonia em organização e comprometimento infeccioso e/ou neoplásico pulmonar se faz sempre necessário, uma vez que as características clínicas e radiológicas destas lesões são muito semelhantes.

Um total de 14 entre os 38 pacientes tinham associações causais evidentes. Apenas em cinco desses pacientes a condição causal era uma infecção documentada $(S$. pneumoniae, $P$. aeruginosa, bacilos gram-negativos não identificados, $\operatorname{HIV}^{(15)}$ e L. pneumophila), mas neste último caso ainda havia a possibilidade de a pneumonia em organização ser secundária à retocolite ulcerativa ou mesmo ao tratamento com sulfassalazina ${ }^{(16)}$. É incerto se o paciente que evoluiu com insuficiência ventilatória era portador de pneumonia em organização idiopática, como já relatado(17), secundária à SDRA, ou como seqüela de radioterapia $^{(18)}$. Três casos foram relacionados com transplantes de órgãos: dois pulmonares ${ }^{(19)}$ e um renal, sendo que neste último as possíveis associações são as drogas imunossupressoras como a ciclosporina ou infecções oportunistas (Tabela 1). Além da presença de co-morbidades implicáveis, esse grupo de pacientes apresentou menor incidência relativa de tosse e maior incidência relativa de infiltração difusa bilateral.

As provas de função pulmonar demonstraram grande variabilidade de resultados, ao contrário da esperada predominância de um padrão restritivo. Esse achado deve-se provavelmente à grande prevalência de tabagismo e à heterogeneidade dos casos incluídos, muitos deles com lesões localizadas. Os resultados da análise espirométrica de pacientes não fumantes favorecem essa conclusão, pois aí prevalecem os distúrbios restritivos. Muitos pacientes 
tinham diagnóstico clínico de doença pulmonar obstrutiva crônica, o que pode justificar a presença de vários pacientes com distúrbio obstrutivo ou misto. A redução da $\mathrm{DCO}$, que reflete as anormalidades da relação entre ventilação e perfusão decorrentes do preenchimento dos alvéolos, foi sensível, mas não contribuiu de forma marcante no diagnóstico específico.

A análise do subgrupo de pacientes com bronquiolite obliterante associada à pneumonia em organização ao exame anatomopatológico sugere que a presença da mesma estreita o espectro clínico e radiológico encontrado, formando um grupo mais uniforme, a maioria dos pacientes apresentando alterações radiológicas difusas, sintomas e maior proporção de estertores crepitantes ao exame físico, como os pacientes descritos por Epler et al. ${ }^{(4)}$. Na presente série, a maioria desses casos foi processos secundários. Se apenas tais pacientes fossem analisados, entretanto, alguns casos com alterações clínicas e radiológicas semelhantes e compatíveis com a forma idiopática de pneumonia em organização seriam excluídos. Do mesmo modo, dois desses pacientes apresentavam alterações radiológicas localizadas, e um era assintomático, tal como os casos em que apenas pneumonia em organização foi observada.

A presente série descreve um subgrupo de pacientes em que a probabilidade de encontrar bronquiolite obliterante associada à pneumonia em organização é pequena: pacientes assintomáticos com alterações radiológicas em exame ocasional e nenhuma condição clínica predisponente. Nenhum dos oito pacientes estudados com essas características apresentava bronquiolite obliterante.

Os corticosteróides são atualmente os agentes terapêuticos de eleição e foram empregados por períodos que variam de seis a 12 meses na dose inicial de 1-1,5mg/ $\mathrm{kg} / \mathrm{dia}^{(1)}$. A resposta clínica é usualmente rápida, precedendo a melhora radiológica, que pode levar meses. Alguns pacientes necessitam de tratamento de manutenção devido à recorrência; as lesões radiológicas podem não desaparecer completamente. Os fatores de risco associados com pior resposta ao tratamento são a associação com colagenoses ou outras condições predisponentes, o tabagismo, a presença de hipoxemia, ou infiltrado pulmonar difuso como apresentação radiológica ${ }^{(20,21)}$. Pelo fato de tratar-se de uma série retrospectiva, nem todos os pacientes realizaram espirometria com mensuração de todas as variáveis ou tomografia computadorizada do tórax, o que prejudica a análise do grupo como todo e pode ser responsável pela ausência de valor diagnóstico da espirometria. Ainda, nem todos os espécimes pulmonares foram obtidos por toracotomia, considerada atualmente necessária, a não ser em casos típicos.

Em suma, a pneumonia em organização pode ser responsável por uma gama diversa de apresentações clíni- cas e radiológicas. Uma grande parte dos casos encontrados na prática diária são secundários a outras condições, que sempre devem ser pesquisadas no momento do diagnóstico. A presença de sintomas e lesões radiológicas múltiplas aumenta a possibilidade de bronquiolite obliterante ao exame anatomopatológico. Lesões localizadas no paciente assintomático reduzem tal possibilidade e muitos destes casos provavelmente refletem seqüelas de infecções pulmonares prévias clinicamente inaparentes. A forma idiopática pode, entretanto, apresentar-se de ambas as formas. Um número significativo de casos de pneumonia em organização pode ser confundido com carcinoma brônquico e o diagnóstico diferencial baseado apenas em dados radiológicos é inseguro, devendo a pneumonia em organização ser considerada dentre doenças que podem mimetizar neoplasias ou processos infecciosos pulmonares com evolução não usual.

\section{REFERÊNCIAS}

1. Cordier JF. Organizing pneumonia. Thorax 2000;55:318-28.

2. Sulavik SB. The concept of organizing pneumonia. Chest 1989;96:967-8.

3. Rubin AS. Outras pneumopatias intersticiais. In: Corrêa da Silva LC, editor. Condutas em pneumologia. Rio de Janeiro: Revinter.

4. Epler GR, Colby TV, McLoud TC. Bronchiolitis obliterans organizing pneumonia. N Engl J Med 1985;312:152-8.

5. Corrin B. Bronchiolitis obliterans organizing pneumonia: a British view. Chest 1992;102:7S.

6. Mroz BJ, Sexauer WP, Meade A, Balsara G. Hemoptysis as the presenting symptom in bronchiolitis obliterans organizing pneumonia. Chest 1997; 111:1775-8.

7. I Consenso Brasileiro Sobre Espirometria. J Pneumol 1996;22.

8. Izumi T, Masanori K, Nishimura K, Nagai S. Bronchiolitis obliterans organizing pneumonia: clinical features and differential diagnosis. Chest 1992;102:715-9.

9. King TE, Mortenson RL. Cryptogenic organizing pneumonitis: the North American experience. Chest 1992;102:8S

10. Cordier JF, Loire R, Brune J. Idiopathic bronchiolitis obliterans organizing pneumonia: definition of clinical profiles in a series of 16 patients. Chest 1989;96:999-1004.

11. Bellomo R, Finlay M, McLaughlin P, Tai E. Clinical spectrum of cryptogenic organising pneumonitis. Thorax 1991;46:554-8.

12. Müller NL, Staples CA, Miller RR. Bronchiolitis obliterans organizing pneumonia: CT features in 14 patients. Am J Radiol 1990;154:983-7.

13. Kohno N, Ikezoe J, Takaeshi J, Takeushi N. Focal organizing pneumonia: CT appearance. Radiology 1993;189:119-23.

14. Domingo JA, Peréz-Calvo JI, Carretero JA, Ferrando J. Bronchiolitis obliterans organizing pneumonia: an unusual cause of solitary pulmonary nodule. Chest 1993;103:1621-23.

15. Allen J, Wewers M. HIV-associated bronchiolitis obliterans organizing pneumonia. Chest 1989;96:197-8.

16. Hamadeh MA, Atkinson J, Smith L. Sulfasalazine-induced pulmonary disease. Chest 1992;101:1033-7.

17. Nizami IY, Kissner DG, Vissher DW, Dubaybo BA. Idiopathic bronchiolitis obliterans organizing pneumonia: an acute life-threatening syndrome. Chest 1995; 108:271-7

18. Kaufman J, Komorowski R. Bronchiolitis obliterans: a new complication of irradiation pneumonitis. Chest 1990;97:1243-4.

19. Chaparro C, Chanberlain D, Maurer J, Winton Tl. Bronchiolitis obliterans organizing pneumonia (BOOP) in lung transplant recipients. Chest 1996; 100:1150-3.

20. Costabel U, Guzman J, Teschler H. Bronchiolitis obliterans with organizing pneumonia: outcome. Am J Respir Crit Care Med 1994;149:1670-5.

21. Watanabe K, Senju S, Wenfo, Shirakusa T. Factors related to the relapse of bronchiolitis obliterans organizing pneumonia. Chest 1998;114:1599-606. 